\title{
四川盆地中部侏罗-白严系界线的统计分析
}

\author{
耿良玉 \\ （中国科学院南京地质古生物斫究所）
}

1977 年冬, 马其鸿、叶春辉、刘兆生和作者参加了四川盆地中部侏罗-白严系的野外地层 工作. 该项工作由四川省地质局组织, 是 1:20 万地质图的先行工作 (图 1).

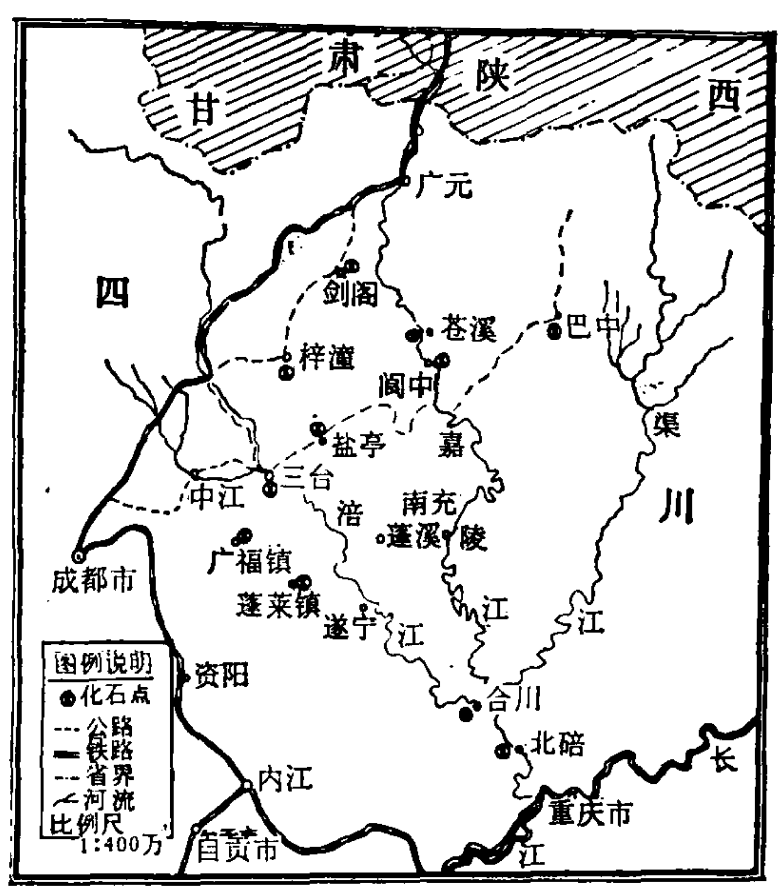

图 1 化石产地图

本区中生代地层分为: 属下白严统的有 梓潼组、柏梓场组、盐亭组,上侏罗统有蓬莱 镇组、遂宁组，中侏罗统有沙溪庙组、新田沟 组、自流井组，下侏罗统有珍珠冲组，上三叠 统有须家河组。以上各组之间均整合接触 (珍珠冲组与须家河组或为假整合接触).

1959 年第一届全国地层会议及 1974 年 西南中生代座谈会均提出：本区侏罗系的上 界, 位于蓬莱镇组之上, 修正了葛利普 (Grabau）等人的老概念 ${ }^{[1]}$. 然而，近年来在莲莱 镇组顶部发现了大量叶肢介(Orthestheria spp. 及 Migransia spp.). 叶肢介工作者将该组肢 解为二: 顶部为下白严统, 其余部分为侏罗 系. 轮藻工作者倾向于认为, 整个莲莱镇组 由于单调而极丰富的 Euaclistochara 这一属的 存在,在时代上相当于甘肃王家山组 (中侏罗 统). 瓣鳃类及介形类工作者认为，应该坚持 1959 及 1974 年的意见. 因而，意见分歧仅在于 蓬莱镇组的时代归宿.

本文试图从统计角度提供一点研究问题的线索. 化石在地层中的分布见表 1 .

应用文献 [2]的方法,我们首先将自流井组、新田沟组作为 I 类,柏梓场组和梓潼组作为 II 类. 这两类分别为侏罗系、白严系, 目前并无争议. 关键在于遂宁组、蓬莱镇组及盐亭组的时 代归属。

首先, 求各组地层与上述两类重心(即平均值)间的欧氏距离

$$
d_{E}=\sqrt{\sum_{i=1}^{21}\left(x_{i j}-x_{i k}\right)^{2}},
$$


表 1

\begin{tabular}{|c|c|c|c|c|c|c|c|c|c|c|c|c|c|c|c|c|c|c|c|c|c|c|c|}
\hline & \multicolumn{2}{|c|}{ 序 } & 1 & 2 & 3 & 4 & 5 & 6 & 7 & 8 & 9 & 10 & 11. & 12 & 13 & 14 & 15 & 16 & 17 & 18 & 19 & 20 & 21 \\
\hline & \multicolumn{2}{|c|}{ 地 ${ }^{\text {石 }}$} & 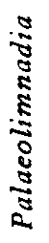 & $\begin{array}{l} \\
8 \\
8 \\
8 \\
5\end{array}$ & 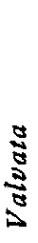 & 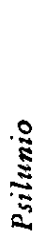 & 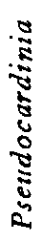 & 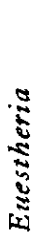 & 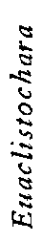 & 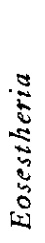 & 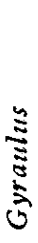 & 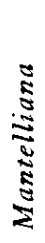 & 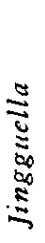 & 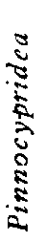 & 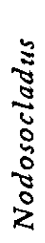 & 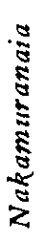 & 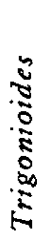 & 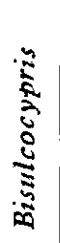 & 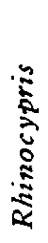 & 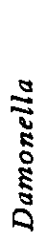 & 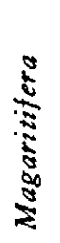 & 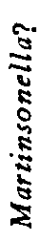 & 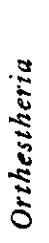 \\
\hline 10 & \multicolumn{2}{|c|}{ 梓治组 } & 0 & 0 & 0 & 0 & 0 & 0 & 0 & 0 & 0 & 0 & 1 & 1 & 0 & 0 & 0 & 0 & 1 & 1 & 0 & 0 & 0 \\
\hline 9 & \multicolumn{2}{|c|}{ 柏梓场组 } & 0 & 0 & 0 & 0 & 0 & 0 & 0 & 0 & 1 & 0 & 1 & 1 & 1 & 1 & 1 & 0 & 1 & 1 & 0 & 0 & 1 \\
\hline 8 & \multicolumn{2}{|c|}{ 盐 亭 组 } & 0 & 0 & 0 & 0 & 0 & 0 & 0 & 0 & 0 & 1 & 1 & 1 & 0 & 0 & 0 & 0 & 1 & 1 & 0 & 0 & 0 \\
\hline 7 & \multicolumn{2}{|c|}{ 莲莱镇组 } & 0 & 0 & 0 & 0 & 0 & 0 & 1 & 1 & 0 & 1 & 0 & 0 & 0 & 0 & 0 & 0 & 0 & 1 & 0 & 1 & 1 \\
\hline 6 & \multicolumn{2}{|c|}{ 遂宁组 } & 0 & 0 & 1 & 0 & 0 & 0 & 1 & 0 & 0 & 0 & 0 & 0 & ) & 0) & 0 & 0 & 0 & 0 & 0 & 0 & 0 \\
\hline 5 & \multicolumn{2}{|c|}{ 沙溪庙组 } & 0 & 0 & 0 & 0 & 1 & 1 & 0 & 0 & 0 & 0 & 0 & 0 & 0 & 0 & 0 & 0 & 0 & 0 & 0 & 0 & 0 \\
\hline 4 & \multicolumn{2}{|c|}{ 新田沟组 } & 0 & 1 & 0 & 1 & 1 & 1 & 0 & 0 & 0 & 0 & 0 & 0 & 0 & 0 & 0 & 0 & 0 & 0 & 0 & 0 & 0 \\
\hline 3 & \multirow{2}{*}{$\begin{array}{l}\text { 自 } \\
\text { 流 } \\
\text { 井 }\end{array}$} & $\begin{array}{l}\text { 大安寨段 } \\
\end{array}$ & 0 & 0 & 0 & 0 & 1 & 0 & 1 & 0 & 0 & 0 & 0 & 0 & 0 & 0 & 0 & 0 & 0 & 0 & 1 & 0 & 0 \\
\hline 2 & & $\begin{array}{l}\text { 므, 鞍 } \\
\text { 山段 }\end{array}$ & 1 & 0 & 0 & 0 & i & 1 & 0 & 0 & 0 & 0 & 0 & 0 & 0 & 0 & 0 & 0 & 0 & 0 & 0 & 0 & 0 \\
\hline 1 & 组 & $\begin{array}{l}\text { 东䚁 } \\
\text { 段 }\end{array}$ & 1 & 0 & 0 & 1 & 1 & 1 & 0 & 0 & 0 & 0 & 0 & 0 & 0 & 0 & 0 & 0 & 0 & 0 & 0 & 0 & 0 \\
\hline
\end{tabular}

其中 $x_{i j}$ 和 $x_{i k}$ 为第 $j$ 组、 $k$ 组的第 $i$ 属化石. 计算结果列为表 2 .

归类的原则是: 若 $d_{E}(\mathbf{x}, \mathrm{I})<d_{E}(\mathbf{x}, \mathrm{II})$, 则向量 $\mathbf{x} \in \mathrm{I}$. 于是得到第一次聚类结果:

类 I: $10 、 9 、 8 ;$ 类 II: $7 、 6 、 5 、 4 、 3 、 2 、 1$.

类 I 中最有代表性的地层是 10 , 类 II 中是 5 . 以 $10 、 5$ 分别作为两类的重心, 重新计算得 第二次聚类:

类 I: $10 、 9 、 8 ;$ 类 II: $7 、 6 、 5 、 4 、 3 、 2 、 1$.

由于相继两次聚类结果无差别,聚类结束. 结论是蓬莱镇组及其以下地层为侏罗系; 盐亭 组及其以上地层为白严系.

这一问题还可由判别分析求解. 我们令白严系 (这里指 9、10) 的综合指数为 1 , 令蓬莱 镇组 (7)、盐亭组 (8) 为待判地层. 若该两组的判别得分大于判别阈值, 归入白严系(K), 小于 判别阈值则归入侏罗系 $(J)$. 计算结果列在表 3 .

判别國值

$$
D_{0}=\frac{0.1904+0.0000}{2}=0.0952,
$$

于是得到如下结果: 蓬莱镇组及其以下地层为侏罗系, 盐亭组及其以上地层为白严系。此结 论与动态聚类分析结论一致。 


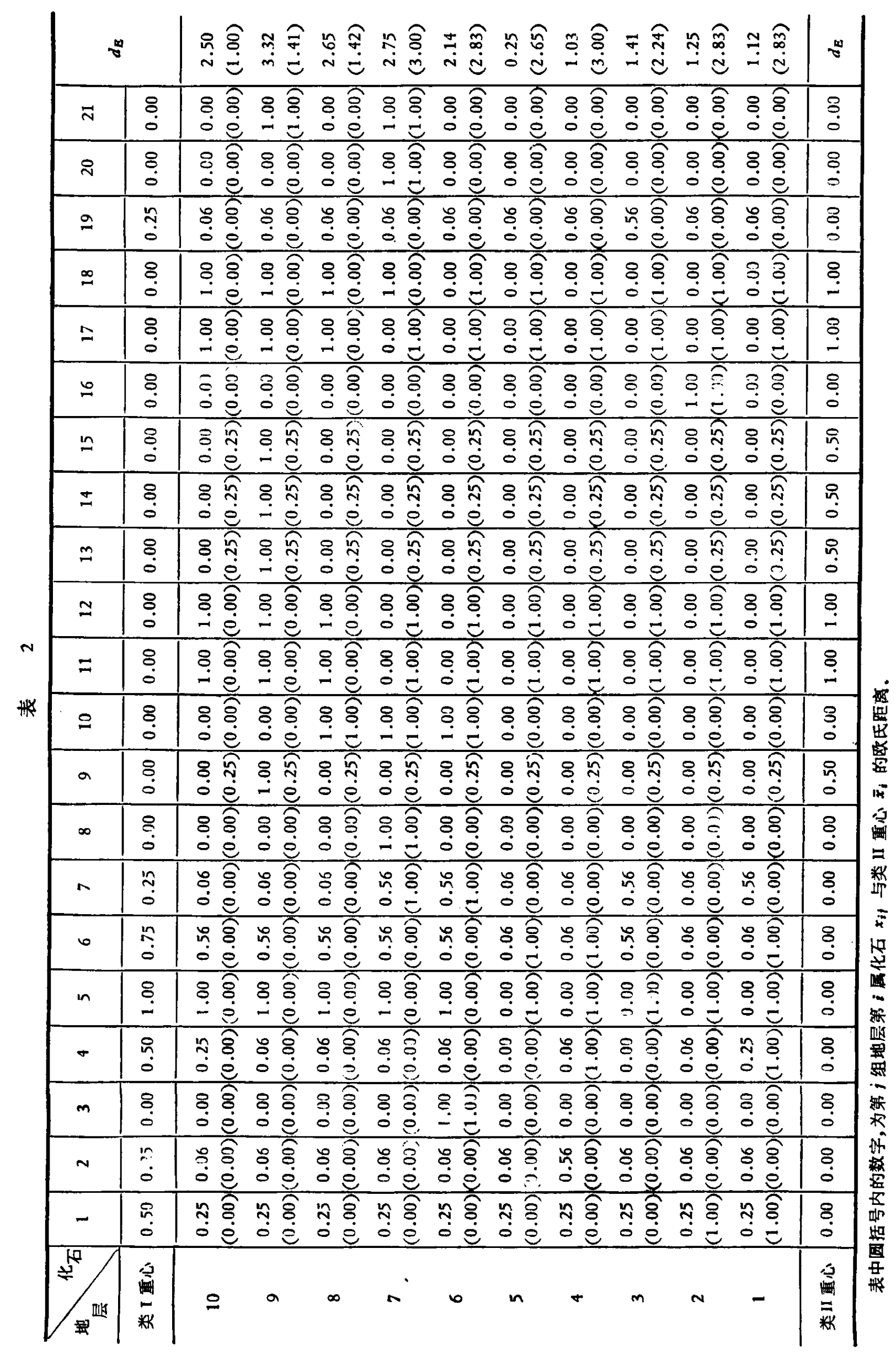


表 3

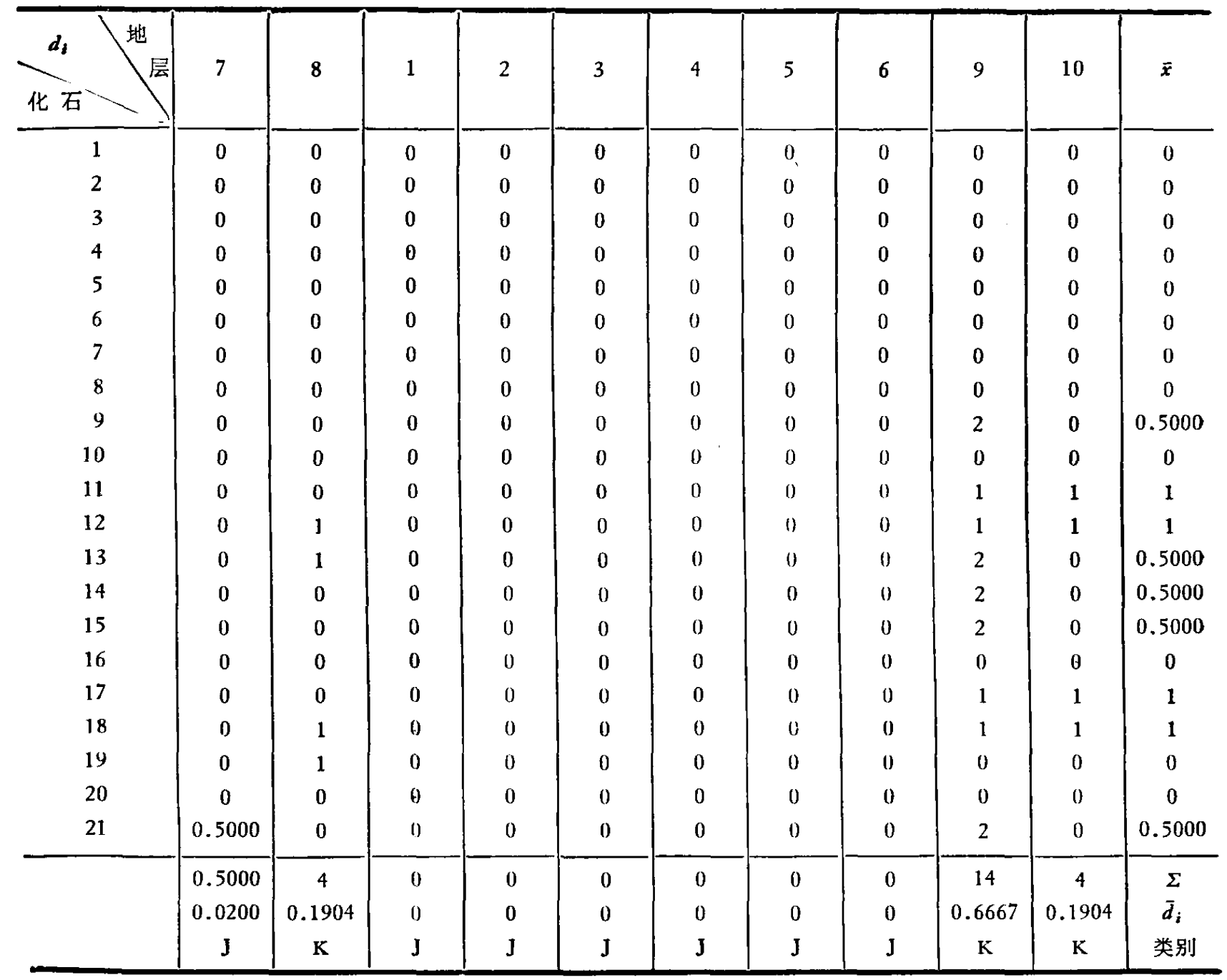

我们可以通过聚类分析对蓬莱镇组以下地层进行划分. 以 Jaccard 相似系数 ${ }^{[3]}$

$$
S_{J}=\frac{a}{a+b+c},
$$

作为刻划地层间相似程度的尺度. 其中 $a$ 为被比较的两组地层中化石间 1-1 匹配数, $b$ 为 0-1 失配数, $c$ 为 1-0 失配数. 该系数的取值域是 $1 \geqslant S_{J} \geqslant 0, S_{J}$ 越大越相似. 计算结果 列在表 4 . 
由于表 4 是一个 $7 \times 7$ 的方阵, 我们只沿主对角线写了一半数据. 由此可得聚类图 (图 3).

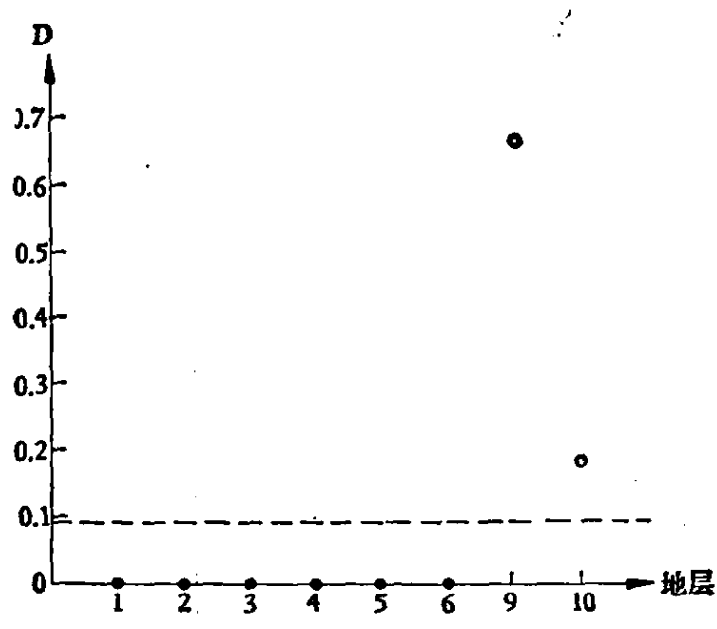

图 2 地层判别图

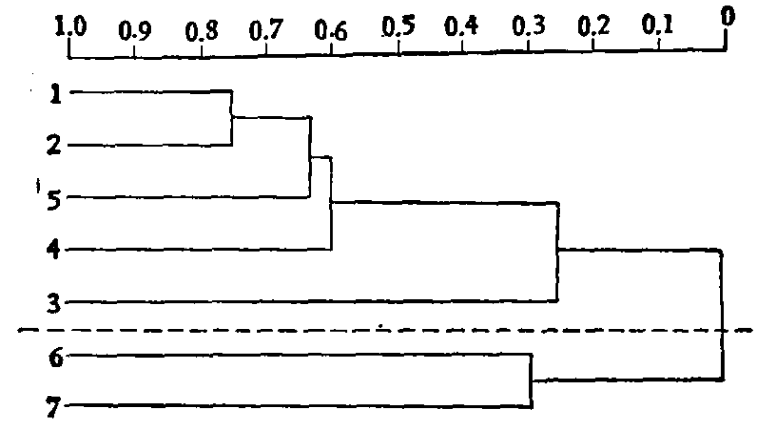

图 3 莲莱镇组以及以下各组聚类图

显然, 自流井组和沙溪庙组为一类 (中侏罗统), 遂宁组和蓬莱镇组为另一类 (上侏罗统). 这样的结论尚待进一步证实.

\title{
今 考 嗝
}

[1] Grabau, A. W., Stratigraphy of China, pt. 2, Mesozoic, 1928, 644-650.

[2] Lefebrre, D. \& David, M., Canadian Journal of Earth Science, 14 (1977), 10: 2232-2245.

[ 3 ] Kaesler, R. L., The Taxonomy, Morphology \& Ecology of Recent Ostracoda, 1969, 21-47.

\section{STATISTICAL ANALYSIS OF JURASSIC-CRETACEOUS BOUNDARY OF CENTRAL SICHUAN BASIN}

\author{
Geng Liang-yu（耿良玉）
}

\begin{abstract}
It is a new subject at present to apply statistical method to dealing with the biostratigraphical problem. With the dynamic elustering and discriminant analysis, the present paper proves that the Jurassic-Cretaceous boundary of the investigated region should be located between the Penglaizhen and Yanting Formations. By applying the cluster analysis, this paper shows that the right boundary of the middle-upper Jurassic in this area should be situated between the Shaximiao and Suining Formations.
\end{abstract}

